\title{
Adaptive finite element simulation of three-dimensional surface tension dominated free-surface flow problems
}

\author{
M. A. Walkley* P. H. Gaskell ${ }^{\dagger} \quad$ P. K. Jimack* \\ M. A. Kelmanson $\quad$ J. L. Summers ${ }^{\dagger}$
}

(Received 3 November 2004, revised 9 April 2005)

\begin{abstract}
An arbitrary Lagrangian-Eulerian finite element method is described for the solution of time-dependent, three-dimensional, freesurface flow problems. Many flows of practical significance involve contact lines, where the free surface meets a solid boundary. This contact line may be pinned to a particular part of the solid but is more typically free to slide in a manner that is characterised by the dynamic contact angle formed by the fluid. We focus on the latter case and use a model that admits spatial variation of the contact angle: thus
\end{abstract}

*School of Computing, University of Leeds, UK. mailto:markw@comp.leeds.ac.uk

$\dagger$ School of Mechanical Engineering, University of Leeds.

$\ddagger$ Department of Applied Mathematics, University of Leeds.

See http://anziamj.austms.org.au/V46/CTAC2004/Walk for this article, (c) Austral. Mathematical Soc. 2005. Published June 29, 2005. ISSN 1446-8735 
permitting variable wetting properties to be simulated. The problems are driven by the motion of the fluid free surface (under the action of surface tension and external forces such as gravity) hence the geometry evolves as part of the solution, and mesh adaptivity is required to maintain the quality of the computational mesh for the physical domain. Continuous mesh adaptivity, in the form of a pseudo-elastic mesh movement scheme, is used to move the interior mesh nodes in response to the motion of the fluid's free surface. Periodic, discrete remeshing stages are also used for cases in which the fluid volume has grown, or is sufficiently distorted, by the free-surface motion. Examples are given of a droplet sliding on an inclined uniform plane and of a droplet spreading on a surface with variable wetting properties.

\section{Contents}

1 Introduction

2 Mathematical model

3 Numerical model

4 Computational examples

5 Conclusions

C568

References

C570

\section{Introduction}

We describe some algorithmic developments, and typical computational results, with an adaptive numerical method for the simulation of viscous, three- 
dimensional, time-dependent, free-surface flow problems [13, 14]. Such problems occur in a wide variety of scientific and engineering applications, such as the spreading of viscous fluids [4], the development and motion of droplets of fluids [10], or in coating flows [7].

In recent years there has been a significant interest in the computational study of such flows using an arbitrary Lagrangian-Eulerian (ALE) finite element methods and it is this approach that is pursued here. Cairncross et al. [3] used a linear hexahedral finite element method to solve the incompressible Navier-Stokes equations and also introduced a dynamic-contactangle model [1] to describe the evolution of a coating flow. Bänsch [2] developed and analysed a tetrahedral Taylor-Hood finite element method for the Navier-Stokes equations. This model included a static contact angle, allowing fluid slip along solid boundaries, but did not account for dynamiccontact-angle effects. Zhou and Derby [15] describe a linear tetrahedral finite element model for the Stokes equations and apply this to the sintering of two spherical particles. Here, as with to Bänsch [2], a three-dimensional incompressible free-surface flow solver based upon the use of implicitly stable elements (the so-called Taylor-Hood element) is developed. These isoparametric elements represent the three-dimensional free surface using piecewise quadratics which is of particular significance when the curvature-dependent surface-tension effects are important.

Perhaps the main limitation of the previous work cited is in restricting the mesh adaptivity to mesh movement techniques alone. In many cases the fluid volume becomes sufficiently distorted, due to the flow, that the computation cannot continue $[1,15]$. Our previous work extended the moving-mesh approach to include discrete remeshing stages at this point, and was applied to a model of the formation of a droplet at the end of a tube [13]. A limitation was that the remeshing stages introduced a small but non-negligible change in fluid volume and, by implication, a change in surface curvature, due to the geometrical representation required for the remeshing. Here the algorithm is improved by incorporating an additional surface projection step 
after discrete remeshing so as to reduce this effect.

Particular attention is also paid to the modelling of the contact line, where the fluid free surface meets a solid surface. For steady flows a given static contact angle between these two surfaces is achieved. This static angle is a physical parameter of the problem and its value is a function of the fluid properties and the solid-surface characteristics. For time-dependent flows a dynamic contact angle model is implemented which allows motion of the free surface along a solid boundary. This dynamic model allows the free surface to maintain contact angles different from the static value while not at equilibrium [14], in line with experimental observations. Specifying a variation in the distribution of the static contact angle allows simulation of problems where the solid surface has preferential wetting areas and, conversely, areas where wetting is inhibited.

\section{Mathematical model}

The class of problems to be considered here is generally characterised by three nondimensional parameters: the Reynolds number, Stokes number and capillary number, respectively

$$
\operatorname{Re}=\frac{\rho L U}{\mu}, \quad \mathrm{St}=\frac{\rho g L^{2}}{\mu U}, \quad \mathrm{Ca}=\frac{\mu U}{\sigma},
$$

written in terms of the fluid density $\rho$, viscosity $\mu$ and surface tension parameter $\sigma$, a characteristic length $L$ and velocity $U$, and gravity $g$. For the purposes of this work flows are considered for which the Reynolds number is small, and the problem to be modelled is described by the threedimensional Stokes equations for velocity field $\mathbf{u}$ and pressure $p$, written in non-dimensional form as

$$
\mathbf{0}=\nabla \cdot \boldsymbol{\sigma}+\operatorname{St} \mathbf{f}, \quad 0=\nabla \cdot \mathbf{u} .
$$


In (2) $\boldsymbol{\sigma}=-p \mathbf{I}+\nabla \mathbf{u}+\nabla \mathbf{u}^{T}$ is the stress tensor and $\mathbf{f}$ is the exterior force. The fluid domain $\Omega$ is assumed to be simply connected and is bounded by either a fluid free surface $\Gamma_{f}$ or a solid wall $\Gamma_{w}$. The contour defined by the interface of these two surfaces is termed the contact line $\gamma_{c}$. On the solid boundary $\Gamma_{w}$ a no-slip condition is applied. On the free surface $\Gamma_{f}$ the following kinematic condition, which describes the evolution of the free surface shape, and normal-stress condition, which includes the effect of surface tension, are applied:

$$
\mathbf{n}_{f} \cdot\left(\mathbf{u}-\dot{\mathbf{x}}_{f}\right)=0, \quad \mathbf{n}_{f} \cdot \boldsymbol{\sigma}=-\mathbf{n}_{f} p_{\mathrm{ext}}+\frac{1}{\mathrm{Ca}}\left(\nabla_{S} \cdot \mathbf{n}_{f}\right) \mathbf{n}_{f} .
$$

In (3) $\mathbf{n}_{f}$ represents the outward normal to the free surface whose location is given by $\mathbf{x}_{f}$, the dot above a variable denotes its time derivative, $\mathbf{u}$ represents the fluid velocity at a point on the free surface, $p_{\text {ext }}$ is the (constant) external pressure, which may be taken as zero for simplicity, and $\nabla_{S}=\left(\mathbf{I}-\mathbf{n}_{f} \mathbf{n}_{f}^{T}\right) \cdot \nabla$ is the surface gradient operator.

For some applications [13] we assume that the dynamic contact line $\gamma_{c}$ is fixed and a no-slip condition applies. In these cases there is, in effect, no restriction on the angle formed between the fluid free surface and the solid boundary. However, in general it is more appropriate to allow the position of the contact line to evolve as part of the flow. It is desirable in these cases to specify a static contact angle, $\theta_{s}$, on this boundary, the value of which is determined a priori by properties of both the fluid and the solid surface, and which is given as a physical parameter of the problem. Furthermore, experiments have shown [5] that, while not at equilibrium, the fluid can support a variable, so-called, dynamic contact angle, $\theta$, that differs from the static value.

Figure 1 depicts the geometry of the contact line on which the model is based. In practice $\mathbf{n}_{f}$ and $\mathbf{n}_{w}$ (the outward normal to the solid boundary) are computed from the current geometry whilst $\mathbf{n}_{c}$, the tangent to the solid surface in the plane of $\mathbf{n}_{f}$ and $\mathbf{n}_{w}$, defines locally the direction in which the free surface is allowed to move. Strictly, this problem cannot be uniquely 


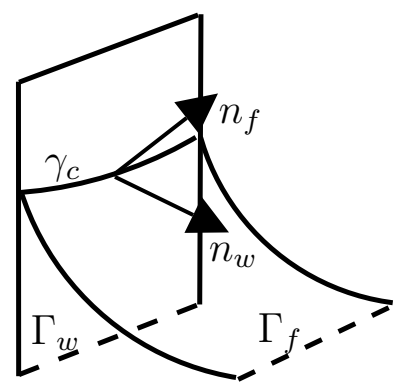

(a) $3 \mathrm{~d}$ model of $\mathbf{n}_{w}$ and $\mathbf{n}_{f}$

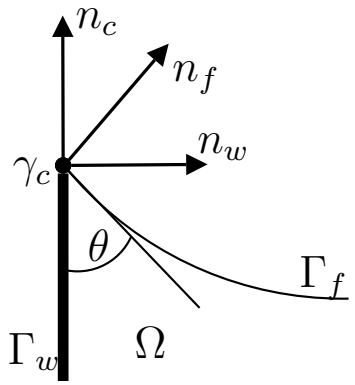

(b) $2 d$ section in the plane

Figure 1: Geometrical description of the dynamic contact line

defined within the Stokes flow framework since the contact line is both part of the solid boundary, which is subject to the no-slip condition, and the fluid boundary, which is subject to the kinematic condition. Detailed mathematical analyses of this problem and alternative mathematical models have appeared $[9,12$, e.g.]. In general these models may be expressed in the form

$$
\mathbf{n}_{w} \cdot \mathbf{n}_{f}=\cos (\theta)=f\left(\theta_{s}, \dot{x}_{c}\right),
$$

with the precise definition of $f\left(\theta_{s}, \dot{x}_{c}\right)$ determined by the selected model. Equation (4) may be used to compute the local speed of the contact line $\dot{x}_{c}$ in the direction $\mathbf{n}_{c}$. The specific model we use here is taken from [5] and was first applied in three dimensions by Baer et al. [1]:

$$
f\left(\theta_{s}, \dot{x}_{c}\right)=\cos \left(\theta_{s}\right)-c_{T} \text { Ca } \dot{x}_{c} .
$$

The constant $c_{T}$ in this model is arbitrary and an appropriate value should be determined empirically to scale the contact line speed relative to the dynamic contact angle. The value $c_{T}=0.99$, used by Baer et al. [1], is taken here. It is straightforward to include this general model of the contact line in the current algorithm, as is described in the following section. 


\section{Numerical model}

In previous work the time-dependent, nonlinear system of equations (2-3) has been solved in a fully coupled fashion [1, e.g.]. This method is computationally expensive due to the size of the nonlinear system since the flow solution and geometry evolution are coupled through the kinematic boundary condition. Here, the computational cost is reduced by decoupling the flow solution from the mesh motion at each time step. The (steady) Stokes equations (2) are used to compute the pressure and velocity field and this is then used to update the free-surface position with the (time-dependent) kinematic boundary condition (3). Interior mesh motion is then performed as a further step.

The Stokes equations are approximated with an isoparametric tetrahedral Taylor-Hood finite element method. This admits a piecewise-quadratic approximation of the fluid geometry allowing an accurate model of the surface curvature and hence the free-surface stress boundary condition. The discretised finite element problem is solved with a preconditioned GMRES iteration using the solution from the previous time step as the initial guess.

The free surface $\Gamma_{f}$ is displaced using an explicit time discretisation of the kinematic condition (3) and the computed velocity field. The dynamic contact line $\gamma_{c}$ is displaced using the chosen contact line model $(4-5)$. The motion of the free surface is driven by the governing equations; however, the interior mesh is then adapted so as to allow the computation to proceed for as long as possible by maintaining a suitable mesh quality. Here, a pseudo-elastic solid motion of the interior mesh points is used, driven by the free-surface boundary displacements. This linear elastic problem is discretised using a linear finite element method and solved with a Gauss-Seidel iterative technique [13]. In practice two iterations are sufficient to produce a satisfactory evolution of the interior mesh.

Discrete remeshing stages are typically required in cases where the fluid 
domain has been distorted by the free-surface motion. Geometric quality measures quantify the volume and surface mesh quality as well as constraints on the minimum and maximum edge lengths allowed in the mesh. The quality of the piecewise-quadratic free-surface mesh is monitored through the integral of the curvature on the mesh edges: $I_{\kappa}=\int_{s}|\kappa| d s$, where $\kappa$ is the curvature [11] computed directly as a piecewise constant on the locally quadratic edge. This measure indicates regions in which surface curvature is large relative to the local mesh resolution and is controlled with a tolerance value. In previous work [14], remeshing the isoparametric, piecewise-quadratic domain introduced a small but non-negligible volume change; in practice it was an order of magnitude greater than the volume change from the mesh movement alone.

To remedy this a further step is now added in which the free-surface nodes from the remeshed domain are projected onto the piecewise-quadratic surface of the original geometry. A geometric search of the original domain locates the neighbouring element for each node in the new computational domain. The isoparametric coordinates of the new node, with respect to the original element, are computed through the solution of a $3 \times 3$ nonlinear system of equations at each node. Only two iterations are required to converge. The free-surface node is then projected onto the original free-surface shape. Since the domain is piecewise quadratic the original free-surface shape cannot be exactly recovered; however, the effect is to reduce the volume change due to complete remeshing to a comparable level to that due to the mesh movement alone. The curvature of the free surface is critical to the computation of the surface-tension forces hence the improvement in the algorithm also minimises any jump in surface pressure that may occur due to the remeshing of the domain. The first example in Section 4 illustrates the improved performance due to this additional stage in the discrete remeshing algorithm. 


\section{Computational examples}

The range of capillary number and Stokes number applicable to this model is derived from the fluid data presented by Martinez [6]. Choosing a length scale $L$ of the order of $L_{c}$, termed the capillary length, where $L_{c}^{2}=\frac{\sigma}{\rho g}$, and a velocity scale $U=\frac{\sigma}{\mu}$, fluid data for pure glycerin, for example, results in $\mathrm{Re} \ll 1$ with Ca and St of order 1, which is within the Stokes flow regime.

For the computations considered in this section, meshing of the initial fluid domain and discrete remeshing stages are carried out using the NETGEN software package [8].

The first example that we present considers the evolution of a droplet sliding on a plane inclined at $20^{\circ}$ to the horizontal. This problem was previously modelled [14]; however, the improvements to the discrete remeshing algorithm described in Section 3 are demonstrated by considering the problem again. The droplet is initially hemispherical with radius 1 and the computational mesh has 396 nodes and 179 elements. The constant time step is $10^{-3}$. The Stokes number and capillary number are both 1 , and the static contact angle $\theta_{s}=90^{\circ}$. Figures 2(a)-(b) show statistics for 20 time units of the droplet simulation for the original algorithm (old) [14] and the improved algorithm (new) described in the previous section. The old and new algorithms introduce volume changes of $3.1 \times 10^{-2}$ (1.5\% of the fluid volume) and $4.6 \times 10^{-4}(0.02 \%)$ respectively at the first remeshing stage. This is comparable to the volume change of $1.9 \times 10^{-4}$ introduced by the explicit update of the free-surface at every time step, up to that point. After 20,000 time steps the fluid volume is now conserved to within $0.1 \%$ of its original value, compared to only $4 \%$ previously. An additional benefit is that remeshing is triggered less frequently, only twice over the course of the new simulation.

As discussed in Section 2, the static contact angle is a function of both the fluid and solid properties; hence a predefined distribution of values are used to simulate areas of preferential wetting. The next example comprises 


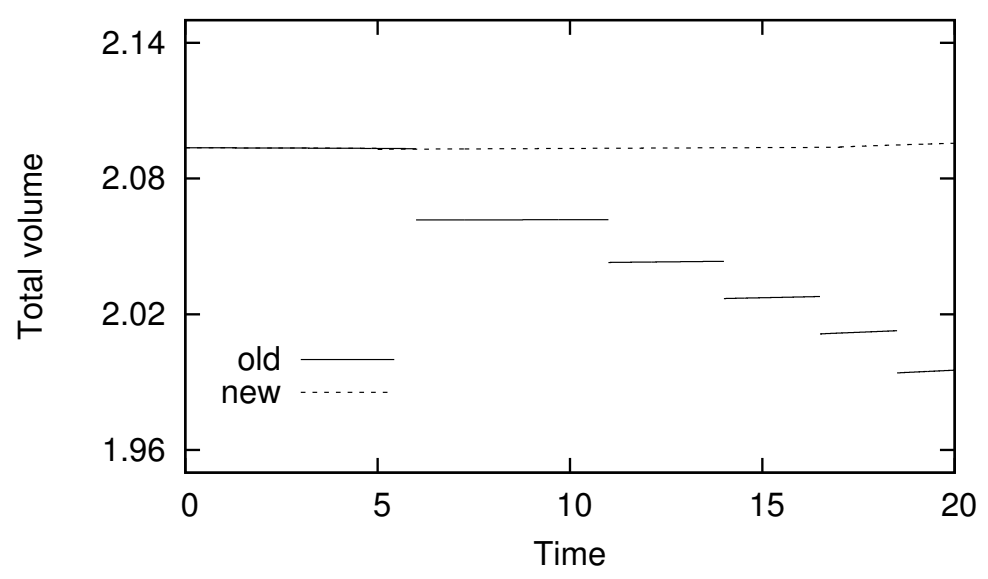

(a)

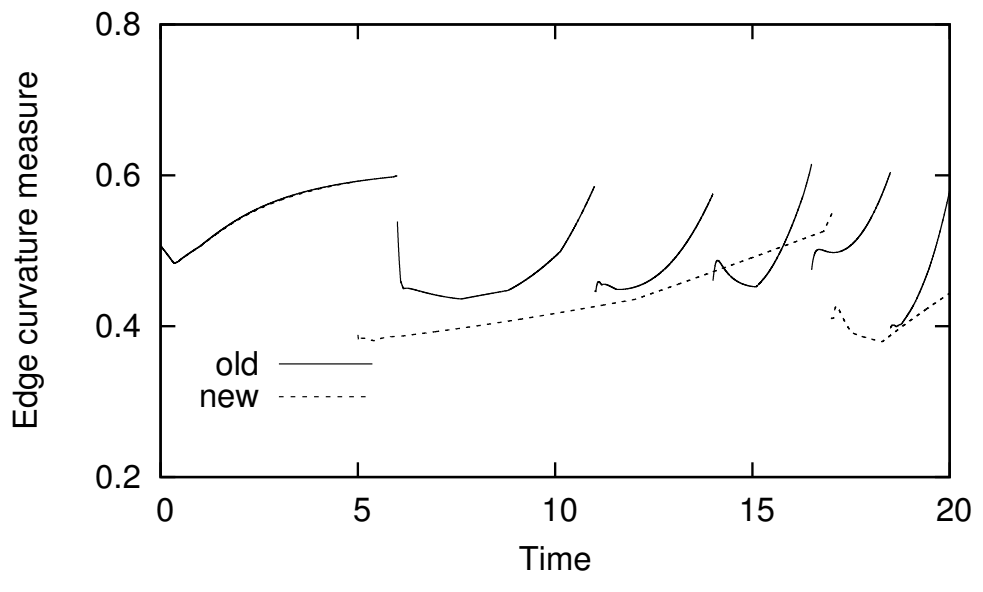

Figure 2: Droplet sliding on an inclined plane: (a) Fluid volume; (b) Edge curvature measure. 
a hemispherical droplet of nondimensional radius 1 with $\mathrm{Ca}=1$ and $\mathrm{St}=4$. The static contact angle is defined as $2 \pi / 3$ for $x<-1$ or $y<-1$ (depicted as the shaded area in Figure 3 ) and to be $\pi / 2$ elsewhere. The initial domain has 2141 nodes and 1159 elements, and a constant time step of $10^{-3}$ is used. Figures 3(a)-(c) show the droplet at three instants during the simulation. The droplet spreads under the action of gravity but is inhibited from moving into the shaded area by the larger static contact angle. The problem is remeshed twice during this simulation.

\section{Conclusions}

An adaptive ALE finite element method for the solution of three-dimensional moving-boundary problems in the presence of dynamic contact lines has been described. In particular, when considering the implementation at the contact line, the piecewise-quadratic model accurately represents the required contact angles and surface curvature at the solid boundary. The mathematical model of the contact line is quite general and alternative models $[9,12]$ are possible within the framework of equations $(4-5)$ which should be investigated and contrasted. At present the model is limited to Stokes flow and further work is required to extend the model to the Navier-Stokes regime, which would allow a wider range of practical applications to be addressed.

Acknowledgment: We thank the EPSRC for funding this work through grant GR/R25453/01. 
(a) $\mathrm{t}=0.0$

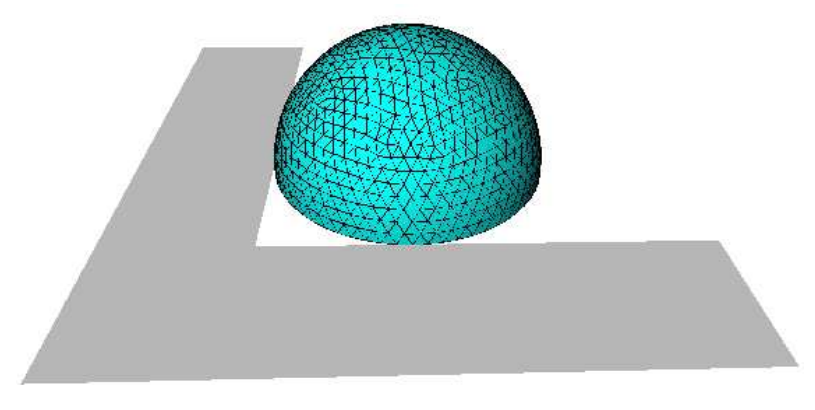

(b) $\mathrm{t}=4.0$

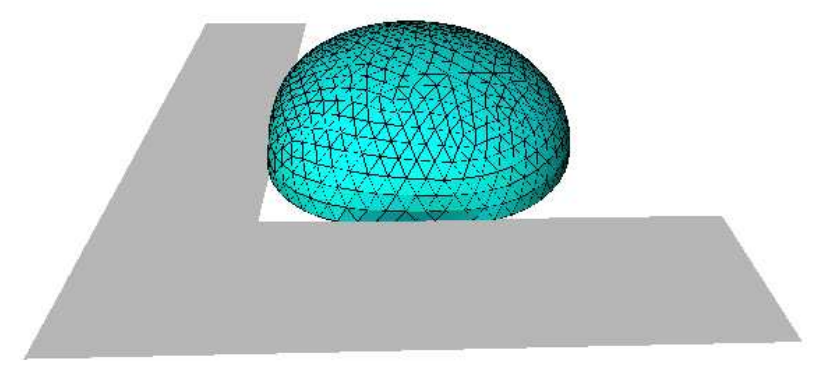

(c) $\mathrm{t}=20.0$

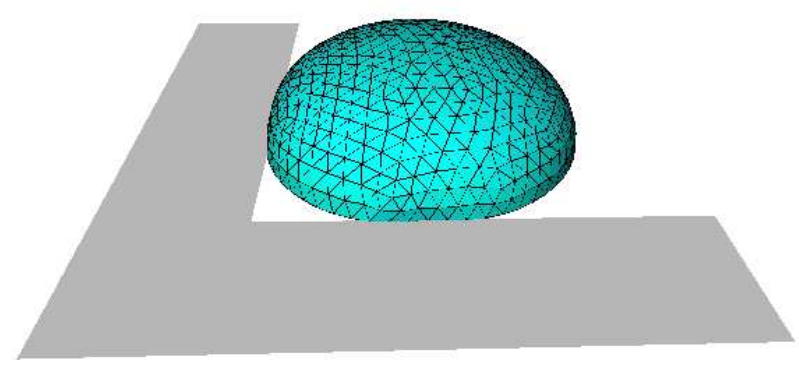

Figure 3: Droplet spreading on a patterned surface 


\section{References}

[1] T. A. Baer, R. A. Cairncross, P. R. Schunk, R. R. Rao, P. A. Sackinger. A finite element method for free surface flows of incompressible fluids in three dimensions. Part II. Dynamic wetting lines. International Journal for Numerical Methods in Fluids. 33:405-427, 2000. C560, C563, C564

[2] E. Bänsch. Finite element discretization of the Navier-Stokes equations with a free capillary surface. Numerische Mathematik. 88:203-235, 2001. C560

[3] R. A. Cairncross, P. R. Schunk, T. A. Baer, R. R. Rao, P. A. Sackinger. A finite element method for free surface flows of incompressible fluids in three dimensions. Part I. Boundary fitted mesh motion. International Journal for Numerical Methods in Fluids. 33:375-403, 2000. C560

[4] P. H. Gaskell, P. K. Jimack, M. Sellier and H. M. Thompson. Efficient and accurate time adaptive multigrid simulations of droplet spreading. International Journal for Numerical Methods in Fluids. 45:1161-1186, 2004. C560

[5] S. F. Kistler. Hydrodynamics of wetting. In J. C. Berg, editor, Wettability, pages 311-429. M. Dekker: New York, 1993. C562, C563

[6] I. Martinez. http://imartinez.etsin.upm.es/dat1/eLIQ.htm. 2004. C566

[7] H. Saito and L. E. Scriven. Study of coating flow by the finite element method. Journal of Computational Physics. 42:53-76, 1981. C560

[8] J. Schöberl. NETGEN - An advancing front 2D/3D-mesh generator based on abstract rules. Computing and Visualization in Science. 1:41-52, 1997. C566 
[9] Y. D. Shikhmurzaev. Moving contact lines in liquid/liquid/solid systems. Journal of Fluid Mechanics. 334:211-249, 1997. C563, C568

[10] H. A. Stone. Dynamics of drop deformation and breakup in viscous fluids. Annual Review of Fluid Mechanics. 26:65-102, 1994. C560

[11] D. J. Struik. Differential Geometry (2nd edn). Addison-Wesley, 1961. C565

[12] O. V. Voinov. Wetting: inverse dynamic problem and equations for microscopic particles. Journal of Colloid and Interface Science. 226:5-15, 2000. C563, C568

[13] M. A. Walkley, P. H. Gaskell, P. K. Jimack, M. A. Kelmanson and J. L. Summers. Finite element simulation of three-dimensional free-surface flow problems. Journal of Scientific Computing (To appear). C560, C562, C564

[14] M. A. Walkley, P. H. Gaskell, P. K. Jimack, M. A. Kelmanson and J. L. Summers. Finite element simulation of three-dimensional free-surface flow problems with dynamic contact lines. International Journal for Numerical Methods in Fluids. 47:1353-1359, 2005. C560, C561, C565, C566

[15] H. Zhou, J. J. Derby. An assessment of a parallel, finite element method for 3D, moving-boundary flows driven by capillarity for simulation of viscous sintering. International Journal for Numerical Methods in Fluids. 36:841-865, 2001. C560 